\title{
THE ACTION OF WEAK ACIDS ON SOLUBLE FLUORIDES.
}

By P. A. ELLIS RICHARDS, F.I.C.

(Read at the Meeting, May 6, 1914.)

THE fluorides of the alkali metals have recently come into prominence as suggested preservatives for articles of food.

In Reports to the Local Government Board by Drs. MacFadden and MonierWilliams (Food Reports No. 17 and Report on the Work of Inspectors of Foods for the year 1912-13) reference is made to one or two preparations of this nature advertised under proprietary names, with the statement that the preservative is of a perfectly harmless character.

As, so far, these preservatives have been found mainly in milk, cream, and butter, the action, if any, of lactic acid upon soluble fluorides becomes a matter of some importance. With a view to obtaining information on this point, as a preliminary experiment, some pure concentrated lactic acid was allowed to act upon a little 
powdered sodium fluoride contained in a lead capsule covered with a waxed glass marked in the usual way. Evolution of hydrofluoric acid speedily occurred, and distinct etching of the glass was obtained, the latter being naturally increased when the vessel was warmed.

Dilute solutions of lactic acid of 5, 2 and 1 per cent. strength respectively were all found to act in a similiar manner upon the fluorides of sodium and potassium, liberating hydrofluoric acid. In the latter, and subsequent experiments, watchglasses waxed and marked on the concave surfaces were employed to hold the mixed solutions.

The positive result obtained with lactic acid suggested that probably other organic acids might act in a similar manner.

The acids most likely to be found in foods-acetic, butyric, tartaric, malic, and citric acids-were experimented with in turn, and in each case, employing a concentration of 1 per cent. of acid and 1 per cent. of sodium fluoride, distinct etching resulted.

I may perhaps mention that all the ucids employed were carefully tested before use to insure their purity as regards traces of mineral acids, whilst both the sodium and potassium fluorides were free from ammonium fluoride. Control experiments of the same duration, employing similar apparatus containing the fluoride solution only, gave no sign of etching.

The ease with which hydrofluoric acid was liberated by the organic acids mentioned suggested the possibility of carbonic acid gas producing the same result. To determine this point, tubes or bottles, waxed and marked internally, were employed to hold the sodium fluoride solutions. The carbon dioxide, after passage through washing-bottles, was allowed to bubble through the liquid from time to time for an hour. Hydrofluoric acid was again liberated, the etching, although definite, being not quite so pronounced as in the previous experiments.

By allowing solution of a fluoride saturated with carbonic acid gas to remain in contact with the glass for some hours a much deeper etching was obtained. Hydrofluoric acid was also liberated by formic, salicylic, and benzoic acids, but not by boric acid. Lactic acid and the other acids mentioned above, that gave positive results with sodium and potassium fluorides, failed to do so with calcium fluoride, even when the latter was employed in a fine state of subdivision.

Dilute solutions of the mineral acids-sulphuric, nitric, hydrochloric, hydrobromic, and hydriodic acids-as one would expect, all liberated hydrofluoric acid from sodium and potassium fluorides.

The two salts differ in some respects, potassium fluoride being deliquescent, whilst sodium fluoride is a dry powder. Both slowly absorb carbon dioxide from the atmosphere, hydrofluoric acid being set free, and this may possibly account for the action of the fluorides of the alkali metals upon the glass of bottles in which they have been long stored.

As a result of these experiments it is evident that both sodium and potassium fluorides are easily decomposed by the majority of weak acids with which they may be brought in contact, and this fact emphasises their undesirability as preservatives for food of any kind. 


\section{Discussion,}

The President remarked that all the alkaline fluorides were more or less readily hydrolysed in aqueous solution, and presumably any addition of acid would increase the dissociation, so that a little hydrofluoric acid would be formed. This would be driven off on heating, and a further quantity would be produced, and so on. With boric acid a boro-fluoride would probably be formed. On the addition of boric acid to an acid fluoride solution, a strongly alkaline solution was obtained, and the same, of course, happened with silica.

Mr. E. R. Bolton said that the fact that fluorides were very effective in preventing the growth of mould in butter and margarine was no doubt due to formation of hydrofluoric acid in the manner which the President had mentioned, the fluoride being acted upon by the fatty acid resulting from hydrolysis of the fat by the mould.

Dr. LANDER remarked that the liability of fluorides to bydrolytic dissociation furnished an interesting problem in connection with the periodic law, since theoretically one. would anticipate less hydrolysis with fluorides than with chlorides.

Dr. Lessing drew attention to the well-known experiment in which ultramarine was decolorised in a solution of hydrofluoric acid by passing carbonic acid into it.

The President remarked that hydrofluoric acid did not fall into line with the chlorides and bromides, its formula at ordinary temperatures being, not $\mathrm{HF}$, but $\mathrm{H}_{2} \mathrm{~F}_{2}$.

Dr. Monier-Williams said that fluorides had, on the strength of certain experiments on mice and other animals, frequently been stated to be harmless when used as food preservatives. It seemed, however, extremely dangerous to draw such a conclusion in view of certain experiments which had been carried out on the Continent on the amount of fluorine ingested and excreted by dogs fed for a prolonged period on food containing small amounts of sodium fluoride-amounts considerably less than would be required to cause gastric irritation. It would appear that a certain quantity of the fluorine thus ingested remained in the body, associated with, and possibly replacing to a certain extent, the phosphates of the bones and teeth, and that in the course of a year or more comparatively large quantities might thus be taken up. Until these results had been confirmed or disproved, it was obvious that caution must be exercised in drawing conclusions from isolated experiments of a few days' duration upon animals, and that the possibility of sodium fluoride acting as a cumulative poison was by no means excluded.

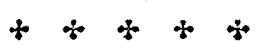

